\title{
On the Ideal Convergence of Double Sequences in Locally Solid Riesz Spaces
}

\author{
A. Alotaibi, ${ }^{1}$ B. Hazarika, ${ }^{2}$ and S. A. Mohiuddine ${ }^{1}$ \\ ${ }^{1}$ Department of Mathematics, Faculty of Science, King Abdulaziz University, P.O. Box 80203, Jeddah 21589, Saudi Arabia \\ ${ }^{2}$ Department of Mathematics, Rajiv Gandhi University, Rono Hills, Doimukh, Arunachal Pradesh 791112, India
}

Correspondence should be addressed to A. Alotaibi; mathker11@hotmail.com

Received 8 December 2013; Accepted 9 March 2014; Published 2 April 2014

Academic Editor: Adem Kilicman

Copyright (C) 2014 A. Alotaibi et al. This is an open access article distributed under the Creative Commons Attribution License, which permits unrestricted use, distribution, and reproduction in any medium, provided the original work is properly cited.

\begin{abstract}
The aim of this paper is to define the notions of ideal convergence, $I$-bounded for double sequences in setting of locally solid Riesz spaces and study some results related to these notions. We also define the notion of $I^{*}$-convergence for double sequences in locally solid Riesz spaces and establish its relationship with ideal convergence.
\end{abstract}

\section{Introduction and Preliminaries}

In 1951, Fast [1] and Steinhaus [2] introduced the concept of statistical convergence for single sequences, independently. Some basic and important properties of this concept were studied by Buck [3], Šalát [4], Schoenberg [5], and Fridy [6]. Later, the notion of statistical convergence for single sequences was further defined in various spaces; see Çakalli and Khan [7-9], Di Maio et al. [10, 11], Hazarika [12-14], Maddox [15], Mohiuddine et al. [16-19], and so forth. Some application of statistical summability methods is presented in $[20,21]$. In 2003, the notion of statistical convergence for single sequences has been extended to double sequences by Mursaleen and Edely [22]. Recently, the statistical convergence and statistical Cauchy for double sequences have been defined in the framework fuzzy and intuitionistic normed spaces by Mohiuddine et al. [23] and Mursaleen and Mohiuddine [24], respectively, and established some interesting results related to the concept of statistical convergence and statistical Cauchy double sequences. Recently, it was defined and studied by Mohiuddine et al. [25] in the setting of locally solid Riesz spaces while for single sequences this concept was first studied by Albayrak and Pehlivan [26] (also see [27-29]). An application of locally solid Riesz spaces in economics can be found in [30].

The notion of ideal convergence for single sequences, which is a generalization of the concept of statistical convergence, was first defined and studied by Kostyrko et al. [31]. Let us recall the notion of ideal convergence and related concepts by Kostyrko et al. [31] as follows. Let $\mathbb{N}$ be a nonempty set. Then a family of sets $I \subseteq P(\mathbb{N})$ (power set of $\mathbb{N}$ ) is said to be an ideal if $I$ is additive; that is, $A, B \in I \Rightarrow A \cup B \in I$ and $A \in I, B \subseteq A \Rightarrow B \in I$. A family of sets $I \subset P(\mathbb{N})$ (power sets of $\mathbb{N}$ ) is called an ideal if and only if, for each $A, B \in I$, we have $A \cup B \in I$ and, for each $A \in I$ and each $B \subset A$, we have $B \in I$. A nonempty family of sets $\mathscr{F} \subset P(\mathbb{N})$ is a filter on $\mathbb{N}$ if and only if $\Phi \notin \mathscr{F}$; for each $A, B \in \mathscr{F}$, we have $A \cap B \in \mathscr{F}$ and for each $A \in \mathscr{F}$ and each $A \subset B$, we have $B \in \mathscr{F}$. An ideal $I$ is called nontrivial ideal if $I \neq \Phi$ and $\mathbb{N} \notin I$. Clearly $I \subset P(\mathbb{N})$ is a nontrivial ideal if and only if $\mathscr{F}=\mathscr{F}(I)=\{\mathbb{N}-A: A \in I\}$ is a filter on $\mathbb{N}$. A nontrivial ideal $I \subset P(\mathbb{N})$ is called admissible if and only if $\{\{x\}: x \in \mathbb{N}\} \subset I$. A nontrivial ideal $I$ is maximal if there cannot exist any nontrivial ideal $J \neq I$ containing $I$ as a subset.

We remark that if $I=I_{f}=\{A \subseteq \mathbb{N}: A$ is a finite subset $\}$, then the corresponding convergence coincides with the usual convergence. Also, if $I=I_{\delta}=\{A \subseteq \mathbb{N}: \delta(A)=0\}$, then the corresponding convergence coincides with the statistical convergence (where $\delta(A)$ denotes the natural density of the set $A$ ). In the above cases, both $I_{f}$ and $I_{\delta}$ are nontrivial admissible ideals of $\mathbb{N}$.

Kumar [32] defined the notions of $I$ and $I^{*}$-convergence of double sequence and studied some properties of these notions. Recently, Das et al. [33] introduced the concepts of $I$ and $I^{*}$-convergence of double sequences in the setting of metric space and established some relationship between these 
types of convergence. Quite recently, Mursaleen and Mohiuddine defined and studied the notion of $I$-convergence, $I^{*}$ convergence, $I$-limit points, and $I$-cluster points for single and double sequences, in $[34,35]$, respectively, in probabilistic normed spaces. Şahiner et al. [36] and Gürdal and Açik [37] introduced the notion of ideal convergence and $I$-Cauchy sequence in 2-normed spaces, respectively. Mursaleen and Alotaibi [38] introduced the notion of ideal convergence in random 2-normed spaces and later on it was extended by Mohiuddine et al. [39] from single to double sequences. For more details on these concepts, one can be referred to [40-52].

Now we recall the definition of locally solid Riesz spaces and some related concepts as follows. Let $X$ be a real vector space and let $\leq$ be a partial order on this space. $X$ is said to be an ordered vector space if it satisfies the following properties:

(1) if $x, y \in X$ and $y \leq x$, then $y+z \leq x+z$ for each $z \in X$;

(2) if $x, y \in X$ and $y \leq x$, then $a y \leq a x$ for each $a \geq 0$.

If, in addition, $X$ is a lattice with respect to the partial ordering, then $X$ is said to be a Riesz space (or a vector lattice)(see [53]).

For an element $x$ of a Riesz space $X$, the positive part of $x$ is defined by $x^{+}=x \vee \bar{\theta}=\sup \{x, \bar{\theta}\}$, the negative part of $x$ by $x^{-}=(-x) \vee \bar{\theta}$, and the absolute value of $x$ by $|x|=x \vee(-x)$, where $\bar{\theta}$ is the zero element of $X$.

A subset $S$ of $X$ is said to be solid if $y \in S$ and $|x| \leq|y|$ implies $x \in S$.

A topology $\tau$ on a real vector space $X$ that makes the addition and scalar multiplication continuous is said to be a linear topology, that is, when the mappings

$$
\begin{gathered}
(x, y) \longrightarrow(x+y) \quad(\text { from }(X \times X, \tau \times \tau) \longrightarrow(X, \tau)), \\
(\lambda, x) \longrightarrow(\lambda x) \quad\left(\text { from }\left(\mathbb{R} \times X, \tau^{\prime} \times \tau\right) \longrightarrow(X, \tau)\right)
\end{gathered}
$$

are continuous, where $\tau^{\prime}$ is the usual topology on $\mathbb{R}$. In this case the pair $(X, \tau)$ is called a topological vector space.

Every linear topology $\tau$ on a vector space $X$ has a base $N$ for the neighborhoods of $\bar{\theta}$ satisfying the following properties.

(1) Each $Y \in N$ is a balanced set; that is, $a x \in Y$ holds for all $x \in Y$ and every $a \in \mathbb{R}$ with $|a| \leq 1$.

(2) Each $Y \in N$ is an absorbing set; that is, for every $x \in$ $X$, there exists $a>0$ such that $a x \in Y$.

(3) For each $Y \in N$ there exists some $E \in N$ with $E+E \subseteq$ $Y$.

A linear topology $\tau$ on a Riesz space $X$ is said to be locally solid (see [54]) if $\tau$ has a base at zero consisting of solid sets. A locally solid Riesz space $(X, \tau)$ is a Riesz space $X$ equipped with a locally solid topology $\tau$. For more details on these concepts, one can be referred to [55-57].

Throughout the paper, the symbol $N_{\text {sol }}$ will stand for a base at zero consisting of solid sets and satisfying conditions (1), (2), and (3) in a locally solid topology. Also we assume that $I_{2}$ is a nontrivial admissible ideal of $\mathbb{N} \times \mathbb{N}$.

\section{Ideal Convergence of Double Sequences in LSR-Spaces}

Throughout the paper $X$ will denote the Hausdorff locally solid Riesz space, which satisfies the first axiom of countability. For our convenience, here and in what follows, we will write an LSR-space instead of a locally solid Riesz space.

The notion of convergence for double sequence was first introduced by Pringsheim [58] as follows. We say that a double sequence $x=\left(x_{j, k}\right)_{j, k \in \mathbb{N}}$ of reals is convergent to $L$ in Pringsheim's sense (briefly, $P$-convergent) provided that given $\epsilon>0$ there exists a positive integer $N$ such that $\mid x_{j, k}-$ $L \mid<\epsilon$ whenever $j, k \geq N$.

Let $K \subset \mathbb{N} \times \mathbb{N}$ and $K(m, n)$ denotes the number of $(i, j)$ in $K$ such that $i \leq m$ and $j \leq n$ (see [22]). Then the lower natural density of $K$ is defined by $\underline{\delta}_{2}(K)=\liminf _{m, n \rightarrow \infty}(|K(m, n)| / m n)$. In this case, the sequence $(K(m, n) / m n)$ has a limit in Pringsheim's sense; then we say that $K$ has a double natural density and is defined by $P-\lim _{m, n \rightarrow \infty}(|K(m, n)| / m n)=\delta_{2}(K)$.

In the recent past, Mohiuddine et al. [25] introduced the notion of statistical convergence of double sequences in LSR-space as follows. Let $(X, \tau)$ be a LSR-space. A double sequence $\left(x_{k, l}\right)$ of points in $X$ is said to be $S_{2}(\tau)$-convergent to an element $x_{0}$ if for each $\tau$-neighborhood $V$ of zero

$$
\delta_{2}\left(\left\{(k, l) \in \mathbb{N} \times \mathbb{N}: x_{k, l}-x_{0} \notin V\right\}\right)=0 .
$$

Now we introduce the notions of $I_{2}(\tau)$-convergence and $I_{2}(\tau)$-bounded double sequences in LSR-spaces.

Definition 1. Let $(X, \tau)$ be a LSR-space. A double sequence $\left(x_{k, l}\right)$ of points in $X$ is said to be $I_{2}(\tau)$-convergent to an element $x_{0}$ of $X$ if for each $\tau$-neighborhood $V$ of zero

$$
\left\{(k, l) \in \mathbb{N} \times \mathbb{N}: x_{k, l}-x_{0} \notin V\right\} \in I_{2} .
$$

That is,

$$
\left\{(k, l) \in \mathbb{N} \times \mathbb{N}: x_{k, l}-x_{0} \in V\right\} \in \mathscr{F} .
$$

In this case, one writes $I_{2}(\tau)-\lim _{k, l \rightarrow \infty} x_{k, l}=x_{0}$ or $\left(x_{k, l}\right) \stackrel{I_{2}(\tau)}{\longrightarrow}$ $x_{0}$.

Definition 2. Let $(X, \tau)$ be a LSR-space. Then, a double sequence $\left(x_{k, l}\right)$ of points in $X$ is said to be $I_{2}(\tau)$-bounded in $X$ if, for each $\tau$-neighborhood $V$ of zero, there is some $a>0$,

$$
\left\{(k, l) \in \mathbb{N} \times \mathbb{N}: a x_{k, l} \notin V\right\} \in I_{2} .
$$

Definition 3. Let $(X, \tau)$ be a LSR-space. One says that a double sequence $x=\left(x_{k, l}\right)$ is $I_{2}(\tau)$-Cauchy in $X$ if, for each $\tau$ neighborhood $V$ of zero, there exist $p, q \in \mathbb{N}$ such that, for all $k, m \geq p$ and $l, n \geq q$,

$$
\left\{(k, l) \in \mathbb{N} \times \mathbb{N}: x_{k, l}-x_{m, n} \notin V\right\} \in I_{2} .
$$

Definition 4. Let $(X, \tau)$ be a LSR-space. Then, a double sequence $x=\left(x_{k, l}\right)$ in $X$ is said to be $I_{2}^{*}(\tau)$-convergent to $x_{0}$ if there is a set $K=\{(k, l)\} \subseteq \mathbb{N} \times \mathbb{N}, k, l=1,2, \ldots$, with $K \in F$ such that $\lim _{k, l} x_{k, l}=x_{0}$. In this case, one writes $I_{2}^{*}(\tau)$ $\lim _{k, l} x_{k, l}=x_{0}$. 
Theorem 5. Let $(X, \tau)$ be a LSR-space. Every $I_{2}(\tau)$-convergent sequence in $X$ has only one limit.

Proof. Suppose that $x=\left(x_{k, l}\right)$ is a double sequence in $X$ such that $I_{2}(\tau)-\lim _{k, l} x_{k, l}=x_{0}$ and $I_{2}(\tau)-\lim _{k, l} x_{k, l}=y_{0}$. Let $V$ be any $\tau$-neighborhood of zero. Also for each $\tau$-neighborhood $V$ of zero there is a set $Y \in N_{\text {sol }}$ such that $Y \subseteq V$. Let $W$ in $N_{\text {sol }}$ be such that $W+W \subseteq Y$. We define the sets $A_{1}$ and $A_{2}$ as follows:

$$
\begin{aligned}
& A_{1}=\left\{(k, l) \in \mathbb{N} \times \mathbb{N}: x_{k, l}-x_{0} \in W\right\}, \\
& A_{2}=\left\{(k, l) \in \mathbb{N} \times \mathbb{N}: x_{k, l}-y_{0} \in W\right\} .
\end{aligned}
$$

Since $I_{2}(\tau)-\lim x_{k, l}=x_{0}$ and $I_{2}(\tau)-\lim x_{k, l}=y_{0}$, we get $A_{1}, A_{2} \in \mathscr{F}$. Now, let $A=A_{1} \cap A_{2}$. Then we have

$$
x_{0}-y_{0}=x_{0}-x_{k, l}+x_{k, l}-y_{0} \in W+W \subseteq Y \subseteq V .
$$

As we know, intersection of all $\tau$-neighborhoods $V$ of zero is the singleton set $\{\bar{\theta}\}$ because $(X, \tau)$ is Hausdorff. Hence $x_{0}-$ $y_{0}=0$; that is, $x_{0}=y_{0}$.

Theorem 6. Let $(X, \tau)$ be a LSR-space and let $\left(x_{k, l}\right)$ and $\left(y_{k, l}\right)$ be two double sequences of points in $X$. Then,

(i) if $I_{2}(\tau)-\lim _{k, l} x_{k, l}=x_{0}$ and $I_{2}(\tau)-\lim _{k, l} y_{k, l}=y_{0}$, then $I_{2}(\tau)-\lim _{k, l}\left(x_{k, l}+y_{k, l}\right)=x_{0}+y_{0}$

(ii) if $I_{2}(\tau)-\lim _{k, l} x_{k, l}=x_{0}$, then $I_{2}(\tau)-\lim _{k, l} a x_{k, l}=a x_{0}$ for $a \in \mathbb{R}$.

Proof. Assume that $I_{2}(\tau)-\lim _{k, l} x_{k, l}=x_{0}$ and $I_{2}(\tau)-\lim _{k, l} y_{k, l}=$ $y_{0}$. Suppose that $V$ is an arbitrary $\tau$-neighborhood of zero. Then there exists $Y \in N_{\text {sol }}$ such that $Y \subseteq V$. Let $W \in N_{\text {sol }}$ such that $W+W \subseteq Y$. Thus, we can write

$$
\begin{aligned}
& B_{1}=\left\{(k, l) \in \mathbb{N} \times \mathbb{N}: x_{k, l}-x_{0} \in W\right\}, \\
& B_{2}=\left\{(k, l) \in \mathbb{N} \times \mathbb{N}: y_{k, l}-y_{0} \in W\right\} .
\end{aligned}
$$

Then we have $B_{1}, B_{2} \in \mathscr{F}$.

Let $B=B_{1} \cap B_{2}$. Hence we have $B \in \mathscr{F}$ and

$$
\begin{aligned}
\left(x_{k, l}+y_{k, l}\right)-\left(x_{0}+y_{0}\right)= & \left(x_{k, l}-x_{0}\right) \\
& +\left(y_{k, l}-y_{0}\right) \in W+W \subseteq Y \subseteq V .
\end{aligned}
$$

Therefore

$$
\left\{(k, l) \in \mathbb{N} \times \mathbb{N}:\left(x_{k, l}+y_{k, l}\right)-\left(x_{0}+y_{0}\right) \in V\right\} \in \mathscr{F} .
$$

Since $V$ is arbitrary, we have $I_{2}(\tau)-\lim \left(x_{k, l}+y_{k, l}\right)=x_{0}+y_{0}$.

(ii) Suppose that $I_{2}(\tau)-\lim _{k, l} x_{k, l}=x_{0}$ and also suppose that $V$ is an arbitrary $\tau$-neighborhood of zero. Then there exists $Y \in N_{\text {sol }}$ such that $Y \subseteq V$, so we have

$$
\left\{(k, l) \in \mathbb{N} \times \mathbb{N}: x_{k, l}-x_{0} \in Y\right\} \in \mathscr{F} .
$$

Since $Y$ is balanced, $a\left(x_{k, l}-x_{0}\right) \in Y$ holds for all $x_{k, l}-x_{0} \in Y$ and for every $a \in \mathbb{R}$ with $|a| \leq 1$. Therefore

$$
\begin{aligned}
\{(k, l) & \left.\in \mathbb{N} \times \mathbb{N}: x_{k, l}-x_{0} \in Y\right\} \\
& \subseteq\left\{(k, l) \in \mathbb{N} \times \mathbb{N}: a x_{k, l}-a x_{0} \in Y\right\} \\
& \subseteq\left\{(k, l) \in \mathbb{N} \times \mathbb{N}: x_{k, l}-x_{0} \in V\right\} .
\end{aligned}
$$

Thus, we have

$$
\left\{(k, l) \in \mathbb{N} \times \mathbb{N}: x_{k, l}-x_{0} \in V\right\} \in \mathscr{F}
$$

for each $\tau$-neighborhood $V$ of zero. Now let $|a|>1$ and $[|a|]$ be the smallest integer greater than or equal to $|a|$. Then there exists $W \in N_{\text {sol }}$ such that $[|a|] W \subseteq Y$. From our assumption that $I_{2}(\tau)-\lim _{k, l} x_{k, l}=x_{0}$, we obtain that

$$
K=\left\{(k, l) \in \mathbb{N} \times \mathbb{N}: x_{k, l}-x_{0} \in W\right\} \in \mathscr{F} .
$$

Therefore

$$
\begin{aligned}
\left|a x_{k, l}-a x_{0}\right| & =|a|\left|x_{k, l}-x_{0}\right| \\
& \leq[|a|]\left|x_{k, l}-x_{0}\right| \in[|a|] W \subseteq Y \subseteq V .
\end{aligned}
$$

Since $Y$ is solid, $a x_{k}-a x_{0} \in Y$. It follows that $a x_{k, l}-a x_{0} \in V$. Thus,

$$
\left\{(k, l) \in \mathbb{N} \times \mathbb{N}: a x_{k, l}-a x_{0} \in V\right\} \in \mathscr{F},
$$

for each $\tau$-neighborhood $V$ of zero. We conclude that $I_{2}(\tau)$ $\lim _{k, l} a x_{k, l}=a x_{0}$.

Theorem 7. Let $(X, \tau)$ be a LSR-space. If a double sequence $\left(x_{k, l}\right)$ in $X$ is $I_{2}(\tau)$-convergent, then it is $I_{2}(\tau)$-bounded.

Proof. Assume that $I_{2}(\tau)-\lim _{k, l \rightarrow \infty} x_{k, l}=x_{0}$. Suppose $V$ is an arbitrary $\tau$-neighborhood of zero. Then, there exists $Y \in N_{\text {sol }}$ such that $Y \subseteq V$. Let $W \in N_{\text {sol }}$ such that $W+W \subseteq Y$. Using our assumption, we obtain that

$$
A=\left\{(k, l) \in \mathbb{N} \times \mathbb{N}: x_{k, l}-x_{0} \notin W\right\} \in I_{2} .
$$

Since $W$ is absorbing, there exists $a>0$ such that $a x_{0} \in W$. Let $b$ be such that $|b| \leq 1$ and $b \leq a$. Since $W$ is solid and $\left|b x_{0}\right| \leq\left|a x_{0}\right|$, we have $b x_{0} \in W$. Also, since $W$ is balanced, $x_{k, l}-x_{0} \in W$ implies $b\left(x_{k, l}-x_{0}\right) \in W$. Then we have

$$
\begin{aligned}
b x_{k, l}= & b\left(x_{k, l}-x_{0}\right)+b x_{0} \in W \\
& +W \subseteq V, \quad \text { for each } k, l \in \mathbb{N}-A .
\end{aligned}
$$

Thus

$$
\left\{(k, l) \in \mathbb{N} \times \mathbb{N}: b x_{k, l} \notin W\right\} \in I_{2} .
$$

Hence $\left(x_{k, l}\right)$ is $I_{2}(\tau)$-bounded.

Theorem 8. Let $(X, \tau)$ be a LSR-space and let $\left(x_{k, l}\right),\left(y_{k, l}\right)$, and $\left(z_{k, l}\right)$ be three double sequences of points in $X$ such that

(i) $x_{k, l} \leq y_{k, l} \leq z_{k, l}$, for all $k, l \in \mathbb{N}$,

(ii) $I_{2}(\tau)-\lim _{k, l} x_{k, l}=x_{0}=I_{2}(\tau)-\lim _{k, l} z_{k, l}$.

Then $I_{2}(\tau)-\lim _{k, l} y_{k, l}=x_{0}$.

Proof. Suppose that the given conditions (i) and (ii) hold for the double sequences $\left(x_{k, l}\right),\left(y_{k, l}\right)$, and $\left(z_{k, l}\right)$. Suppose $V$ is an arbitrary $\tau$-neighborhood of zero. Then, there exists $Y \in N_{\text {sol }}$ 
such that $Y \subseteq V$. Let $W \in N_{\text {sol }}$ such that $W+W \subseteq Y$. It follows from (ii) that $P, Q \in F$, where

$$
\begin{aligned}
& P=\left\{(k, l) \in \mathbb{N} \times \mathbb{N}: x_{k, l}-x_{0} \in W\right\}, \\
& Q=\left\{(k, l) \in \mathbb{N} \times \mathbb{N}: z_{k, l}-x_{0} \in W\right\} .
\end{aligned}
$$

Also from the given condition (i), we have

$$
\begin{aligned}
x_{k, l}-x_{0} \leq & y_{k, l}-x_{0} \leq z_{k, l}-x_{0} \\
\Longrightarrow & \left|y_{k, l}-x_{0}\right| \leq\left|x_{k, l}-x_{0}\right| \\
& \quad+\left|z_{k, l}-x_{0}\right| \in W+W \subseteq Y .
\end{aligned}
$$

Since $Y$ is solid, we have $y_{k, l}-x_{0} \in Y \subseteq V$. Thus,

$$
\left\{(k, l) \in \mathbb{N} \times \mathbb{N}: y_{k, l}-x_{0} \in V\right\} \in \mathscr{F},
$$

for each $\tau$-neighborhood $V$ of zero. Thus $I_{2}(\tau)-\lim _{k, l} y_{k, l}=$ $x_{0}$.

Theorem 9. Let $(X, \tau)$ be a LSR-space. A double sequence $\left(x_{k, l}\right)$ is $I_{2}(\tau)$-convergent to $x_{0}$ in $X$ if and only if for each $\tau$ neighborhood $V$ of zero there exists a subsequence $\left(x_{k^{\prime}(r), l^{\prime}(s)}\right)$ of $\left(x_{k, l}\right)$ such that $\lim _{r, s \rightarrow \infty} x_{k^{\prime}(r), l^{\prime}(s)}=x_{0}$ and

$$
\left\{(k, l) \in \mathbb{N} \times \mathbb{N}: x_{k, l}-x_{k^{\prime}(r), l^{\prime}(s)} \notin V\right\} \in I_{2} .
$$

Proof. Suppose that $I_{2}(\tau)-\lim _{k, l \rightarrow \infty} x_{k, l}=x_{0}$. Also, suppose that $V$ is an arbitrary $\tau$-neighborhood of zero. Let $\left\{V_{i}\right\}$ be a sequence of nested base of $\tau$-neighborhoods of zero. For each $i \in \mathbb{N}$, put

$$
E^{(i)}=\left\{(k, l) \in \mathbb{N} \times \mathbb{N}: x_{k, l}-x_{0} \notin V_{i}\right\} .
$$

Then, $E^{(i+1)} \subset E^{(i)}$ and $E^{(i)} \in F$. Let $m(1)$ and $n(1)$ be such that $r>m(1)$ and $s>n(1)$, respectively. Then $E^{(1)} \neq \phi$. For $r, s \in \mathbb{N}$ such that $m(1) \leq r<m(2)$ and $n(1) \leq s<n(2)$, choose $k^{\prime}(r), l^{\prime}(s) \in E^{(i)}$; that is, $x_{k^{\prime}(r), l^{\prime}(s)}-x_{0} \in V_{1}$. In general, choose $m(p+1)>m(p)$ and $n(p+1)>n(p)$ such that $r>m(p+1)$ and $s>n(p+1)$ hold. Then $E^{(p+1)} \neq \phi$. Therefore for all $r, s$ which satisfy $m(p) \leq r<m(p+1)$ and $n(p) \leq s<n(p+1)$, choose $k^{\prime}(r), l^{\prime}(s) \in E^{(p)}$; that is, $x_{k^{\prime}(r), l^{\prime}(s)}-x_{0} \in V_{p}$. Hence, it follows that $\lim _{r, s} x_{k^{\prime}(r), l^{\prime}(s)}=x_{0}$.

Since $V$ is an arbitrary $\tau$-neighborhood of zero, there exists $Y \in N_{\text {sol }}$ such that $Y \subseteq V$. Let $W \in N_{\text {sol }}$ such that $W+W \subseteq Y$. Now

$$
\begin{aligned}
x_{k, l}-x_{k^{\prime}(r), l^{\prime}(s)}= & x_{k, l}-x_{0}+x_{k^{\prime}(r), l^{\prime}(s)} \\
& -x_{0} \in W+W \subseteq Y \subseteq V .
\end{aligned}
$$

Also $I_{2}(\tau)-\lim _{k, l \rightarrow \infty} x_{k, l}=x_{0}$ and $\lim _{r \rightarrow \infty} x_{k^{\prime}(r), l^{\prime}(s)}=x_{0}$ imply that

$$
\left\{(k, l) \in \mathbb{N} \times \mathbb{N}: x_{k, l}-x_{k^{\prime}(r), l^{\prime}(s)} \notin V\right\} \in I_{2} .
$$

Next suppose for an arbitrary $\tau$-neighborhood $V$ of zero that there exists a subsequence $\left(x_{k^{\prime}(r), l^{\prime}(s)}\right)$ of $\left(x_{k, l}\right)$ such that $\lim _{r, s \rightarrow \infty} x_{k^{\prime}(r), l^{\prime}(s)}=x_{0}$ and

$$
\left\{(k, l) \in \mathbb{N} \times \mathbb{N}: x_{k, l}-x_{k^{\prime}(r), l^{\prime}(s)} \notin V\right\} \in I_{2} .
$$

Since $V$ is any $\tau$-neighborhood of zero, we choose $W \in N_{\text {sol }}$ such that $W+W \subseteq V$. Then we have

$$
\begin{aligned}
x_{k, l}-x_{0}= & x_{k, l}-x_{k^{\prime}(r), l^{\prime}(s)} \\
& +x_{k^{\prime}(r), l^{\prime}(s)}-x_{0} \in W+W \subseteq V .
\end{aligned}
$$

That is,

$$
\begin{aligned}
\{(k, l) & \left.\in \mathbb{N} \times \mathbb{N}: x_{k, l}-x_{0} \notin V\right\} \\
& \subseteq\left\{(k, l) \in \mathbb{N} \times \mathbb{N}: x_{k, l}-x_{k^{\prime}(r)} \notin W\right\} \\
\cup & \left\{(r, s) \in \mathbb{N} \times \mathbb{N}: x_{k^{\prime}(r), l^{\prime}(s)}-x_{0} \notin W\right\} .
\end{aligned}
$$

Therefore

$$
\left\{(k, l) \in \mathbb{N} \times \mathbb{N}: x_{k, l}-x_{0} \notin V\right\} \in I_{2} .
$$

Theorem 10. If $\lim _{k, l \rightarrow \infty} x_{k, l}=x_{0}$ and $I_{2}(\tau)-\lim _{k, l \rightarrow \infty} y_{k, l}=$ 0 , then $I_{2}(\tau)-\lim _{k, l \rightarrow \infty}\left(x_{k, l}+y_{k, l}\right)=\lim _{k, l \rightarrow \infty} x_{k, l}$.

Proof. Let $V$ be any $\tau$-neighborhood of 0 . Then there exists $Y \in N_{\text {sol }}$ such that $Y \subseteq V$. Let $W \in N_{\text {sol }}$ such that $W+W \subseteq Y$. Since $\lim _{k, l \rightarrow \infty} x_{k, l}=x_{0}$, then there exist integers $n_{0}, m_{0}$ such that $k \geq n_{0}, l \geq m_{0}$ implies that $x_{k, l}-x_{0} \in W$. Hence

$$
\left\{(k, l) \in \mathbb{N} \times \mathbb{N}: x_{k, l}-x_{0} \notin W\right\} \subseteq \mathbb{N} \times \mathbb{N}-\left\{\left(n_{0}, m_{0}\right)\right\} .
$$

By the assumption $I_{2}(\tau)-\lim _{k, l \rightarrow \infty} y_{k, l}=0,\{(k, l) \in \mathbb{N} \times \mathbb{N}$ : $\left.y_{k, l} \notin W\right\} \in I_{2}$. Thus

$$
\begin{aligned}
\{(k, l) & \left.\in \mathbb{N} \times \mathbb{N}:\left(x_{k, l}-x_{0}\right)+y_{k, l} \notin V\right\} \\
& \subseteq\left\{(k, l) \in \mathbb{N} \times \mathbb{N}: x_{k, l}-x_{0} \notin W\right\} \\
\cup & \left\{(k, l) \in \mathbb{N} \times \mathbb{N}: y_{k, l} \notin W\right\} .
\end{aligned}
$$

That is,

$$
\left\{(k, l) \in \mathbb{N} \times \mathbb{N}:\left(x_{k, l}-x_{0}\right)+y_{k, l} \notin V\right\} \in I_{2} .
$$

This implies that $I_{2}(\tau)-\lim _{k, l \rightarrow \infty}\left(x_{k, l}+y_{k, l}\right)=\lim _{k, l \rightarrow \infty} x_{k, l}$.

Theorem 11. Let $(X, \tau)$ be a LSR-space and let $x=\left(x_{k, l}\right)$ be a double sequence in $X$. If there is a $I_{2}(\tau)$-convergent sequence $y=\left(y_{k, l}\right)$ in $X$ such that $\left\{(k, l) \in \mathbb{N} \times \mathbb{N}: y_{k, l} \neq x_{k, l} \notin V\right\} \in I_{2}$ then $x$ is also $I_{2}(\tau)$-convergent.

Proof. Suppose that $\left\{(k, l) \in \mathbb{N} \times \mathbb{N}: y_{k, l} \neq x_{k, l} \notin V\right\} \in I_{2}$ and $I_{2}(\tau)-\lim _{k, l} y_{k, l}=x_{0}$. Then for an arbitrary $\tau$-neighborhood $V$ of zero, we have

$$
\left\{(k, l) \in \mathbb{N} \times \mathbb{N}: y_{k, l}-x_{0} \notin V\right\} \in I_{2} .
$$

Now,

$$
\begin{aligned}
\{(k, l) & \left.\in \mathbb{N} \times \mathbb{N}: x_{k, l}-x_{0} \notin V\right\} \\
& \subseteq\left\{(k, l) \in \mathbb{N} \times \mathbb{N}: y_{k, l} \neq x_{k, l} \notin V\right\} \\
& \cup\left\{(k, l) \in \mathbb{N} \times \mathbb{N}: y_{k, l}-x_{0} \notin V\right\} .
\end{aligned}
$$


Therefore, we have

$$
\left\{(k, l) \in \mathbb{N} \times \mathbb{N}: x_{k, l}-x_{0} \notin V\right\} \in I_{2} .
$$

Theorem 12. Let $(X, \tau)$ be a LSR-space. If a double sequence $x=\left(x_{k, l}\right)$ is $I_{2}^{*}(\tau)$-convergent to $x_{0}$, then it is $I_{2}(\tau)$-convergent to $x_{0}$.

Proof. Suppose that $I_{2}^{*}(\tau)-\lim _{k} x_{k, l}=x_{0}$. Let $V$ be an arbitrary $\tau$-neighborhood $V$ of zero. Since $I_{2}^{*}(\tau)$ - $\lim _{k, l} x_{k, l}=x_{0}$, there is a set $K=\{(k, l)\} \subseteq \mathbb{N} \times \mathbb{N},(k, l \in \mathbb{N})$ with $K \in F$ such that $k \geq n, l \geq m$ and $(k, l) \in K$ implies $x_{k, l}-x_{0} \in V$. Then

$$
\begin{aligned}
K_{1} & =\left\{(k, l) \in \mathbb{N} \times \mathbb{N}: x_{k, l}-x_{0} \notin V\right\} \\
& \subseteq \mathbb{N} \times \mathbb{N}-\left\{\left(k_{n+1}, l_{m+1}\right),\left(k_{n+2}, l_{m+2}\right), \ldots\right\} .
\end{aligned}
$$

Therefore

$$
K_{1} \in I_{2}
$$

Hence $x$ is $I_{2}(\tau)$-convergent to $x_{0}$.

Theorem 13. The sequential method $I_{2}(\tau)$ is regular.

Proof of the theorem is straightforward, so it is omitted.

From Theorem 12, we can easily obtain the following useful result.

Theorem 14. The sequential method $I_{2}(\tau)$ is subsequential.

\section{Conflict of Interests}

The authors declare that there is no conflict of interests regarding the publication of this paper.

\section{Acknowledgment}

The authors gratefully acknowledge the financial support from King Abdulaziz University, Jeddah, Saudi Arabia.

\section{References}

[1] H. Fast, "Sur la convergence statistique," Colloquium Mathematicum, vol. 2, pp. 241-244, 1951.

[2] H. Steinhaus, "Sur la convergence ordinate et la convergence asymptotique," Colloquium Mathematicum, vol. 2, pp. 73-84, 1951.

[3] R. C. Buck, "Generalized asymptotic density," American Journal of Mathematics, vol. 75, pp. 335-346, 1953.

[4] T. Šalát, "On statistical convergence of real numbers," Mathematica Slovaca, vol. 30, pp. 139-150, 1980.

[5] I. J. Schoenberg, "The integrability of certain functions and related summability methods," The American Mathematical Monthly, vol. 66, pp. 361-375, 1959.

[6] J. A. Fridy, "On statistical convergence," Analysis, vol. 5, no. 4, pp. 301-313, 1985.

[7] H. Çakalli, "Lacunary statistical convergence in topological groups," Indian Journal of Pure and Applied Mathematics, vol. 26, no. 2, pp. 113-119, 1995.
[8] H. Çakalli, "On statistical convergence in topological groups," Pure and Applied Mathematika Sciences, vol. 43, no. 1-2, pp. 2731, 1996.

[9] H. Çakalli and M. K. Khan, "Summability in topological spaces," Applied Mathematics Letters, vol. 24, no. 3, pp. 348-352, 2011.

[10] G. Di Maio and L. D. R. Kočinac, "Statistical convergence in topology," Topology and Its Applications, vol. 156, no. 1, pp. $28-$ 45, 2008.

[11] A. Caserta, G. Di Maio, and L. D. R. Kočinac, "Statistical convergence in function spaces," Abstract and Applied Analysis, vol. 2011, Article ID 420419, 11 pages, 2011.

[12] B. Hazarika, "On ideal convergence in topological groups," Scientia Magna, vol. 7, no. 4, pp. 80-86, 2011.

[13] B. Hazarika, "On generalized difference ideal convergence in random 2-normed spaces," Filomat, vol. 26, no. 6, pp. 1273-1282, 2012.

[14] B. Hazarika, "Lacunary generalized difference statistical convergence in random 2-normed spaces," Proyecciones. Journal of Mathematics, vol. 31, no. 4, pp. 373-390, 2012.

[15] I. J. Maddox, "Statistical convergence in a locally convex space," Mathematical Proceedings of the Cambridge Philosophical Society, vol. 104, no. 1, pp. 141-145, 1988.

[16] S. A. Mohiuddine and M. Aiyub, "Lacunary statistical convergence in random 2-normed spaces," Applied Mathematics \& Information Sciences, vol. 6, no. 3, pp. 581-585, 2012.

[17] S. A. Mohiuddine, H. Şevli, and M. Cancan, "Statistical convergence in fuzzy 2-normed space," Journal of Computational Analysis and Applications, vol. 12, no. 4, pp. 787-798, 2010.

[18] S. A. Mohiuddine, A. Alotaibi, and M. Mursaleen, "A new variant of statistical convergence," Journal of Inequalities and Applications, vol. 2013, article 309, 8 pages, 2013.

[19] E. Savaş and S. A. Mohiuddine, " $\bar{\lambda}$-statistically convergent double sequences in probabilistic normed spaces," Mathematica Slovaca, vol. 62, no. 1, pp. 99-108, 2012.

[20] C. Belen and S. A. Mohiuddine, "Generalized weighted statistical convergence and application," Applied Mathematics and Computation, vol. 219, no. 18, pp. 9821-9826, 2013.

[21] N. L. Braha, H. M. Srivastava, and S. A. Mohiuddine, "A Korovkin's type approximation theorem for periodic functions via the statistical summability of the generalized de la Vallée Poussin mean," Applied Mathematics and Computation, vol. 228, pp. 162-169, 2014.

[22] Mursaleen and O. H. H. Edely, "Statistical convergence of double sequences," Journal of Mathematical Analysis and Applications, vol. 288, no. 1, pp. 223-231, 2003.

[23] S. A. Mohiuddine, H. Şevli, and M. Cancan, "Statistical convergence of double sequences in fuzzy normed spaces," Filomat, vol. 26, no. 4, pp. 673-681, 2012.

[24] M. Mursaleen and S. A. Mohiuddine, "Statistical convergence of double sequences in intuitionistic fuzzy normed spaces," Chaos, Solitons \& Fractals, vol. 41, no. 5, pp. 2414-2421, 2009.

[25] S. A. Mohiuddine, A. Alotaibi, and M. Mursaleen, "Statistical convergence of double sequences in locally solid Riesz spaces," Abstract and Applied Analysis, vol. 2012, Article ID 719729, 9 pages, 2012.

[26] H. Albayrak and S. Pehlivan, "Statistical convergence and statistical continuity on locally solid Riesz spaces," Topology and Its Applications, vol. 159, no. 7, pp. 1887-1893, 2012.

[27] S. A. Mohiuddine, B. Hazarika, and A. Alotaibi, "Double lacunary density and some inclusion results in locally solid 
Riesz spaces," Abstract and Applied Analysis, vol. 2013, Article ID 507962, 8 pages, 2013.

[28] S. A. Mohiuddine and A. Alotaibi, "Statistical summability of double sequences through de la Vallée-Poussin mean in probabilistic normed spaces," Abstract and Applied Analysis, vol. 2013, Article ID 215612, 5 pages, 2013.

[29] S. A. Mohiuddine and M. A. Alghamdi, "Statistical summability through a lacunary sequence in locally solid Riesz spaces," Journal of Inequalities and Applications, vol. 2012, article 225, 9 pages, 2012.

[30] C. D. Aliprantis and O. Burkinshaw, Locally solid Riesz spaces with Applications to Economics, vol. 105 of Mathematical Surveys and Monographs, American Mathematical Society, Providence, RI, USA, 2nd edition, 2003.

[31] P. Kostyrko, T. Šalát, and W. Wilczyński, “ $\mathscr{I}$-convergence," Real Analysis Exchange, vol. 26, no. 2, pp. 669-685, 2000-2001.

[32] V. Kumar, "On $I$ and $I^{*}$-convergence of double sequences," Mathematical Communications, vol. 12, no. 2, pp. 171-181, 2007.

[33] P. Das, P. Kostyrko, W. Wilczyński, and P. Malik, "I and $I^{*}$ convergence of double sequences," Mathematica Slovaca, vol. 58 , no. 5, pp. 605-620, 2008.

[34] M. Mursaleen and S. A. Mohiuddine, "On ideal convergence in probabilistic normed spaces," Mathematica Slovaca, vol. 62, no. 1, pp. 49-62, 2012.

[35] M. Mursaleen and S. A. Mohiuddine, "On ideal convergence of double sequences in probabilistic normed spaces," Mathematical Reports, vol. 12(62), no. 4, pp. 359-371, 2010.

[36] A. Şahiner, M. Gürdal, S. Saltan, and H. Gunawan, "Ideal convergence in 2-normed spaces," Taiwanese Journal of Mathematics, vol. 11, no. 5, pp. 1477-1484, 2007.

[37] M. Gürdal and I. Açık, "On I-Cauchy sequences in 2-normed spaces," Mathematical Inequalities \& Applications, vol. 11, no. 2, pp. 349-354, 2008.

[38] M. Mursaleen and A. Alotaibi, "On I-convergence in random 2-normed spaces," Mathematica Slovaca, vol. 61, no. 6, pp. 933940, 2011.

[39] S. A. Mohiuddine, A. Alotaibi, and S. M. Alsulami, "Ideal convergence of double sequences in random 2-normed spaces," Advances in Difference Equations, vol. 2012, article 149, 8 pages, 2012.

[40] H. Çakalli and B. Hazarika, "Ideal quasi-Cauchy sequences," Journal of Inequalities and Applications, vol. 2012, article 234, 11 pages, 2012.

[41] E. Dündar, Ö. Talo, and F. Başar, "Regularly $\left(\mathscr{I}_{2}, \mathscr{I}\right)$ convergence and regularly $\left(\mathscr{I}_{2}, \mathscr{I}\right)$-Cauchy double sequences of fuzzy numbers," International Journal of Analysis, vol. 2013, Article ID 749684, 7 pages, 2013.

[42] B. Hazarika and S. A. Mohiuddine, "Ideal convergence of random variables," Journal of Function Spaces and Applications, vol. 2013, Article ID 148249, 7 pages, 2013.

[43] B. K. Lahiri and P. Das, " $I$ and $I^{*}$-convergence in topological spaces," Mathematica Bohemica, vol. 130, no. 2, pp. 153-160, 2005.

[44] H. I. Miller, "A measure theoretical subsequence characterization of statistical convergence," Transactions of the American Mathematical Society, vol. 347, no. 5, pp. 1811-1819, 1995.

[45] M. Mursaleen, S. A. Mohiuddine, and O. H. H. Edely, "On the ideal convergence of double sequences in intuitionistic fuzzy normed spaces," Computers \& Mathematics with Applications, vol. 59, no. 2, pp. 603-611, 2010.
[46] F. Nuray and W. H. Ruckle, "Generalized statistical convergence and convergence free spaces," Journal of Mathematical Analysis and Applications, vol. 245, no. 2, pp. 513-527, 2000.

[47] T. Šalát, B. C. Tripathy, and M. Ziman, "On some properties of $I$ convergence," Tatra Mountains Mathematical Publications, vol. 28, pp. 279-286, 2004.

[48] T. Šalát, B. C. Tripathy, and M. Ziman, "On I-convergence field," Italian Journal of Pure and Applied Mathematics, no. 17, pp. 4554, 2005.

[49] B. C. Tripathy and B. Hazarika, " $I$-monotonic and $I$-convergent sequences," Kyungpook Mathematical Journal, vol. 51, no. 2, pp. 233-239, 2011.

[50] B. C. Tripathy and B. Hazarika, "I-convergent sequence spaces associated with multiplier sequences," Mathematical Inequalities \& Applications, vol. 11, no. 3, pp. 543-548, 2008.

[51] B. Tripathy and B. C. Tripathy, "On I-convergent double sequences," Soochow Journal of Mathematics, vol. 31, no. 4, pp. 549-560, 2005.

[52] U. Yamanc1 and M. Gürdal, " $\mathscr{J}^{\mathscr{K}}$-convergence in the topology induced by random 2-normed spaces," International Journal of Analysis, vol. 2013, Article ID 451762, 7 pages, 2013.

[53] A. C. Zaanen, Introduction to Operator Theory in Riesz Spaces, Springer, Berlin, Germany, 1997.

[54] G. T. Roberts, “Topologies in vector lattices," Mathematical Proceedings of the Cambridge Philosophical Society, vol. 48, pp. 533-546, 1952.

[55] L. V. Kantorovich, "Lineare halbgeordnete Raume," Recreational Mathematics, vol. 2, pp. 121-168, 1937.

[56] W. A. J. Luxemburg and A. C. Zaanen, Riesz Spaces I, NorthHolland, Amsterdam, The Netherlands, 1971.

[57] F. Riesz, "Sur la décomposition des opérations fonctionelles linéaires," in Atti Del Congr. Internaz. Dei Mat., 3, Bologna, 1928, pp. 143-148, Zanichelli, 1930.

[58] A. Pringsheim, "Zur Theorie der zweifach unendlichen Zahlenfolgen," Mathematische Annalen, vol. 53, no. 3, pp. 289-321, 1900. 


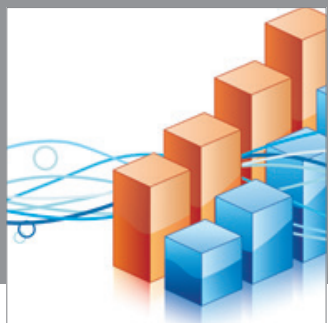

Advances in

Operations Research

mansans

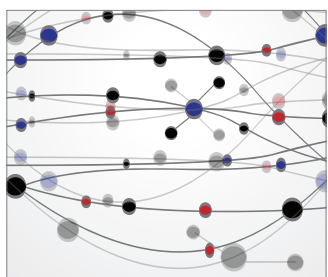

The Scientific World Journal
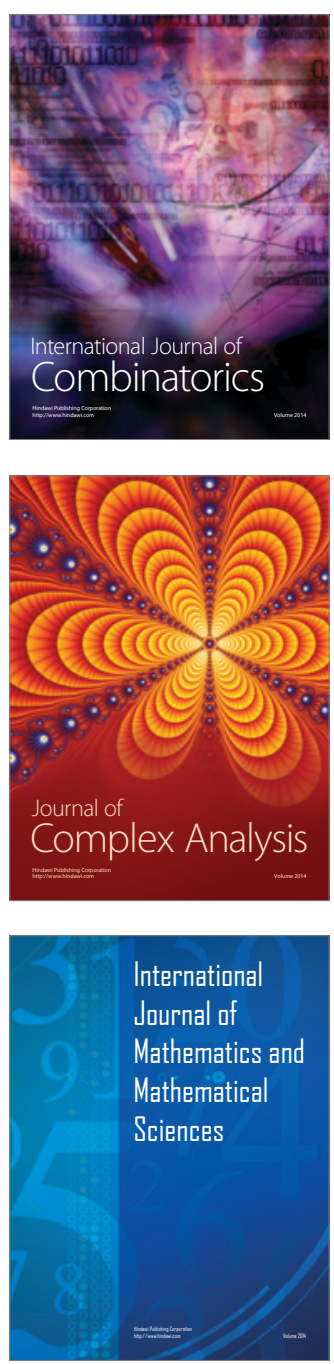
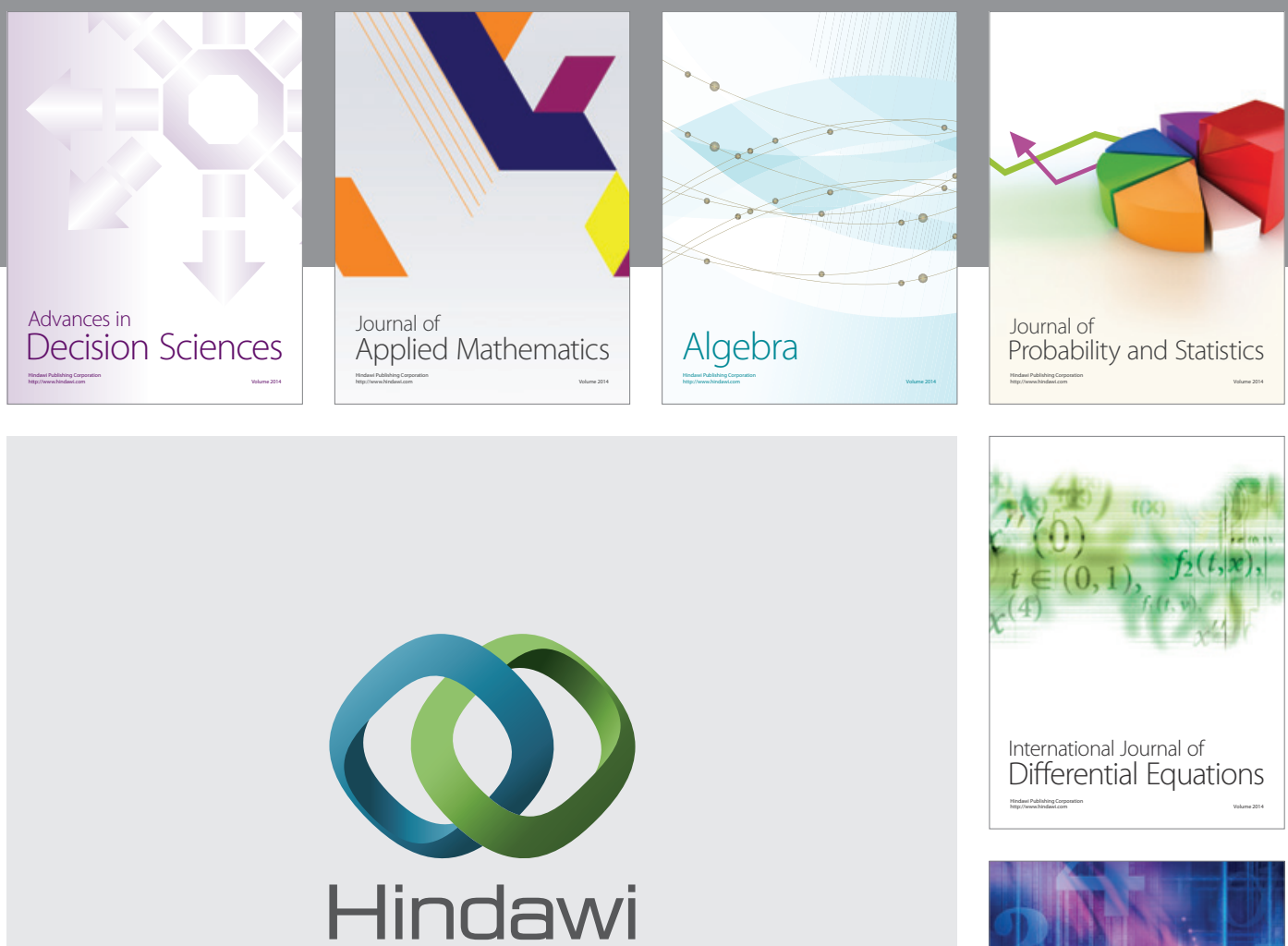

Submit your manuscripts at http://www.hindawi.com
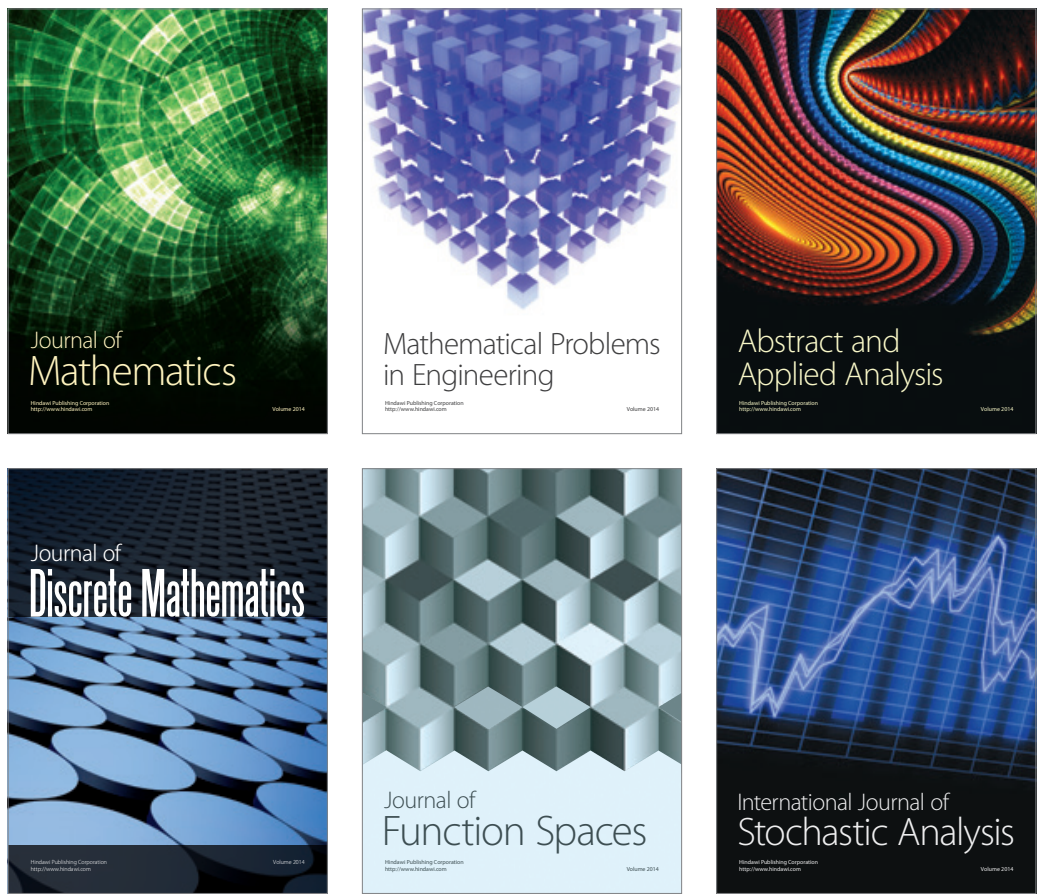

Journal of

Function Spaces

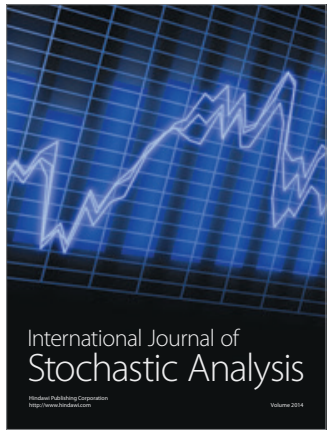

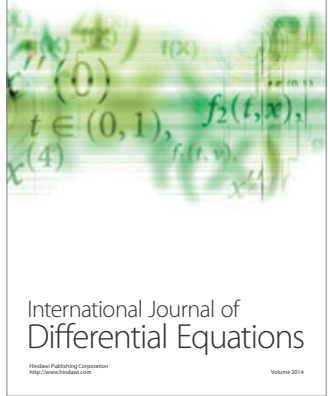
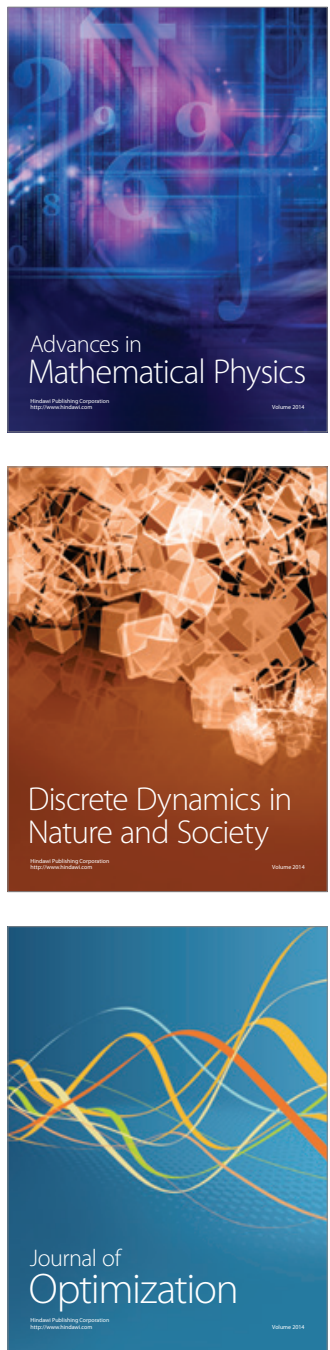